\title{
L3/Lhx8 is a pivotal factor for cholinergic differentiation of murine embryonic stem cells
}

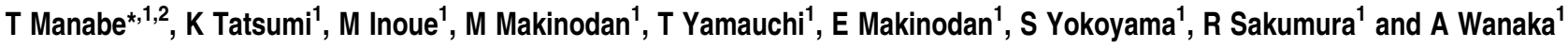

L3/Lhx8 is a member of the LIM-homeobox gene family. Previously, we demonstrated that L3/Lhx8-null mice specifically lacked cholinergic neurons in the basal forebrain. In the present study, we conditionally suppressed L3/Lhx8 function during retinoic acid-induced neural differentiation of a murine embryonic stem (ES) cell line using an L3/Lhx8-targeted small interfering RNA (siRNA) produced by an $\mathrm{H1} .2$ promoter-driven vector. Our culture conditions induced efficient differentiation of the ES cells into neurons and astrocytes, but far less efficient differentiation into oligodendrocytes. Suppression of L3/Lhx8 expression by siRNA led to a dramatic decrease in the number of cells positive for the cholinergic marker ChAT, and overexpression of L3/Lhx8 recovered this effect. However, no significant changes were observed in the number of Tuj $1+$ neurons and GABA + cells. These results strongly suggest that $L 3 / L h \times 8$ is a key factor in the cholinergic differentiation of murine ES cells and is involved in basal forebrain development.

Cell Death and Differentiation (2007) 14, 1080-1085. doi:10.1038/sj.cdd.4402106; published online 23 February 2007

Recently, L3/Lhx8 was identified as a member of the LIM homeobox gene family and is selectively expressed in the medial ganglionic eminence (MGE). ${ }^{1}$ Previously, we generated mice with disrupted expression of the $L 3 / L h \times 8$ gene and reported that $L 3 /$ Lhx8-null mice had a clearly visible phenotype; homozygous mice had a cleft palate. ${ }^{2}$ In addition, we reported that L3/Lhx8-null mice had lost a significant number of basal forebrain cholinergic neurons, while an L3/Lhx8knockdown cell line specifically lacked the cholinergic phenotype. $^{2,3}$ Intriguingly, GABAergic differentiation was increased in the differentiating L3/Lhx8-knockdown N2a cells. ${ }^{3}$ In the basal forebrain, cholinergic and GABAergic neurons mediate the majority of excitatory and inhibitory neurotransmission, respectively. The differentiation mechanisms of cholinergic neurons are of special interest because an abnormality of the cholinergic projection neurons in the basal forebrain has been implicated in neurodegenerative diseases such as Alzheimer's disease.,5

Murine embryonic stem (ES) cells can contribute to the formation of all tissues and are an ideal tool for examining differentiation mechanisms in vitro. Recently, effective neurogenesis from ES cells was achieved using an in vitro stromal cell-derived inducing activity (SDIA) method. ${ }^{6}$ Oct3/4, which plays a critical role in maintaining ES cell pluripotency, ${ }^{7}$ can also promote neuroectoderm formation by ES cells when expression is sustained, and this leads to neural differentiation. ${ }^{8}$ Previous reports have demonstrated that retinoic acid (RA) enhances the cholinergic phenotype in various cell lines in which differentiation can be induced. ${ }^{3,9-17}$ A combination of the above-mentioned culture conditions allows us to achieve effective cholinergic differentiation of ES cells.
To explore the role of the $L 3 / L h x 8$ gene, we employed the RA-induced neural differentiation model in ES cells that stably express Oct3/4 (EB5 cells). Here, we report the effects of small interfering RNA (siRNA) against $L 3 / L h x 8$ mRNA on neural differentiation in this system with special reference to the neurotransmitter phenotype.

\section{Results and Discussion}

Our previous study demonstrated that L3/Lhx8-null mice significantly and specifically lacked cholinergic neurons in the MGE and basal forebrain. ${ }^{2}$ Furthermore, cholinergic differentiation was diminished by L3/Lhx8-knockdown in neuroblastoma Neuro2a (N2a) cells. ${ }^{3}$ Intriguingly, the expression of GABAergic markers and the number of GABAergic cells were dramatically increased in differentiating L3/Lhx8-knockdown N2a cells. These results suggest the possibility that L3/Lhx8 is involved in the determination of neurotransmitter phenotypes (GABAergic or cholinergic cell fate) in a population of neurons during basal forebrain development. However, in that experimental system, N2a cells are committed to a neuronal fate, and it is therefore difficult to clarify whether GABAergic differentiation is promoted as a secondary result of the inhibition of cholinergic differentiation following L3/Lhx8knockdown or whether the L3-depletion positively leads cells toward GABAergic differentiation. On the other hand, in an experimental system using ES cells, which are not committed to a neuronal fate, ES cells may differentiate into cells other than GABAergic neurons, such as glial cells. Therefore, we decided to investigate the role of L3/Lhx8 in phenotypespecific differentiation (and/or developmental) steps via a

\footnotetext{
${ }^{1}$ Department of 2nd Anatomy, Faculty of Medicine, Nara Medical University, 840 Shijyo-cho, Kasihara City, Nara 634-8521, Japan

${ }^{*}$ Corresponding author: T Manabe, Department of Neuronal Differentiation and Regeneration, Center for Developmental Biology (CDB), RIKEN Kobe Institute, 2-2-3

Minatojima-Minamimachi, Chuo-ku, Kobe, Hyogo 650-0047, Japan. Tel: 8178306 3100; Fax: 8178306 3089; E-mail: manabe@cdb.riken.jp

${ }^{2}$ Current address: Department of Neural Differentiation and Regeneration, Center for Developmental Biology (CDB), RIKEN Kobe Institute, 2-2-3 Minatojima-

Minamimachi, Chuo-ku, Kobe, Hyogo 650-0047, Japan

Keywords: Lim-homeobox; L3/Lhx8; acetylcholine; GABA; RNAi

Abbreviations: ES, embryonic stem; siRNA, small interfering RNA; MGE, medial ganglionic eminence; SDIA, stromal cell-derived inducing activity

Received 12.5.06; revised 22.12.06; accepted 04.1.07; Edited by L Greene; published online 23.2.07
} 
loss-of-function study of L3/Lhx8 in ES cells in vitro using an RNAi strategy. We used a vector-based RNAi approach to intracellularly produce a short hairpin double-stranded RNA (dsRNA) from a DNA template under the control of the histon $\mathrm{H} 1.2$ promoter. $^{3}$ The $\mathrm{H} 1.2$ promoter in this vector is an engineered inducible promoter containing a tetracycline (Te) operator (TetO1). A clone stably transfected with this vector expresses green fluorescent protein (GFP) under the control of the cytomegalovirus (CMV) promoter in a Te independent manner.

First, we established the conditions for neural differentiation of ES cells to determine the optimal conditions for the RNAi experiments (Figure 1a-d). Our established conditions effectively induced $\mathrm{Tuj}^{+}{ }^{+}$-neural differentiation of ES cells
$(25.6 \pm 2.2 \%, n=3$; Figure $1 \mathrm{c}$ and $\mathrm{m})$. Furthermore, immunostaining for GFAP demonstrated that both trypsin-treated dispersed cells $(55.7 \pm 6.9 \%, n=3$; Figure $1 n)$ and trypsinuntreated spheres (Figure 1j) differentiated into GFAPpositive astrocytes. However, there was no detectable immunoreactivity to CNPase (an oligodendrocyte marker) in differentiated cells, from either dispersed cells or spheres, despite abundant expression of GFP (dispersed cells: Figure $1 \mathrm{k}$ and I; spheres: Figure $1 \mathrm{~g}$ and $\mathrm{h}$ ). The ES cells used in the present study stably express Oct3/4, which promotes neuronal differentiation (neither astrocytes nor oligodendrocytes appear within 10 days of culture) via the SDIA method. ${ }^{8}$ In our study, using a different RA-mediated neural differentiation method, gave rise to a number of GFAP-positive

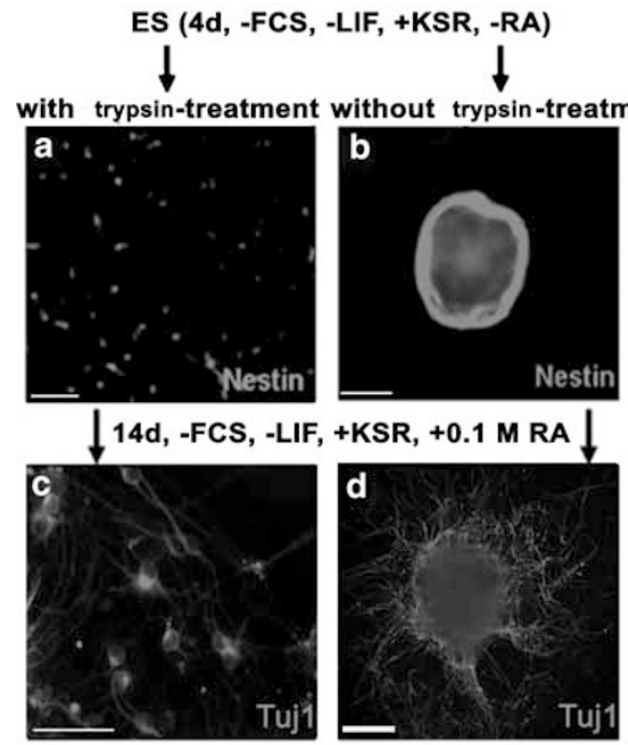

e

RT-PCR

L3 mRNA

with trypsin-treatment without trypsin-treatment
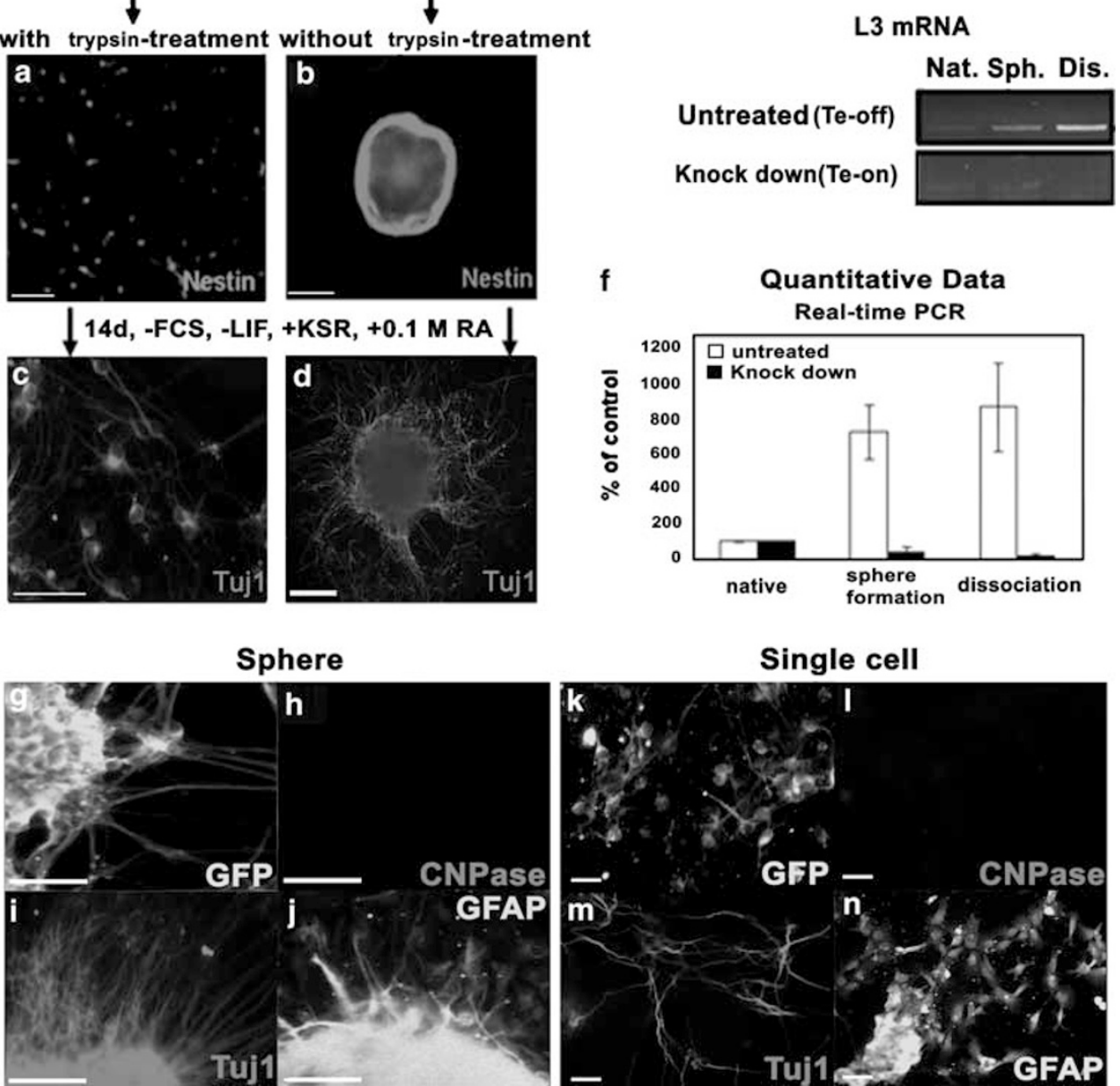

Figure 1 Characterization of the ES cells used in the present study. (a-d) Schematic representation of the strategy for neural differentiation of murine ES cells. Cells cultured under each of the conditions were fixed and immunostained with an anti-Nestin (a, b) or an anti-Tuj1 (c, d) antibody. Scale bar, $50 \mu \mathrm{m}$. (e-f) Selection and characterization of the stable clones used in the present study. (e) Effects of induction of an L3/Lhx8 siRNA on the inhibition of L3/Lhx8 mRNA expression in ES cells. Mock and tetracycline (Te)-inducible L3/Lhx8 siRNA vectors were transfected into ES cells using Targefect F-1, and each clone was picked up after cell selection with hygromycin. The cells were maintained under sphere-formation conditions for 4 days (lane 2, Sph, sphere), followed by specification under differentiation conditions for 14 days (lane 3 , Dis., dissociated) with (e, lower panel, Te-on) or without (e, upper panel, Te-off) Te. Te-untreated (upper panel, Te-off) and Te-treated (lower panel, Te-on) ES cells were collected at each step and total RNA was extracted. RNA was quantified by real-time RT-PCR assays as described in the Materials and Methods. (f) Data show mean \pm S.E. for $n=3$ separate experiments. The Te-induced L3/Lhx8 siRNA efficiently inhibits the L3/Lhx8 mRNA expression underlying the differentiation processes. Native, untreated cells cultured under standard conditions. ( $\mathbf{g}-\mathbf{n})$ Characterization of differentiated ES cells by immunocytochemical analysis. ES cells were maintained in sphere-formation medium (-FCS, - LIF, -RA, + KSR) for 4 days in bacterial-grade dishes, followed by passage on courting dishes with ( $\mathbf{k}-\mathbf{n}$ ) or without (g-j) trypsin treatment. After 14 days, differentiated ES cells were fixed and immunostained with antibodies against GFP $(\mathbf{g}, \mathbf{e})$ or CNPase (k, I) or Tuj1 (i, $\mathbf{m})$ and GFAP (j, n). Scale bar, $20 \mu \mathrm{m}$ 
astrocytes (Figure $1 \mathrm{j}$ and $\mathrm{n}$ ), which is convenient for our purposes, since the cells are not exclusively committed to a neuronal fate.

We obtained stable clones of Te-inducible L3/Lhx8 siRNA-expressing ES cells utilizing an $\mathrm{H} 1.2$ promoter-driven double-stranded hairpin siRNA-producing vector (see Materials and Methods; Figure 1e and f). The vector harbored a CMV promoter-driven GFP cassette, which could label the transfected cells irrespective of the presence or absence of Te. Real-time RT-PCR assays demonstrated that more potent inhibition of L3/Lhx8 expression occurred in the clone under the Te-on condition than the Te-off condition (Figure 1e, lower panel; Figure 1f). However, no significant depletion of L3/Lhx8 mRNA was detected
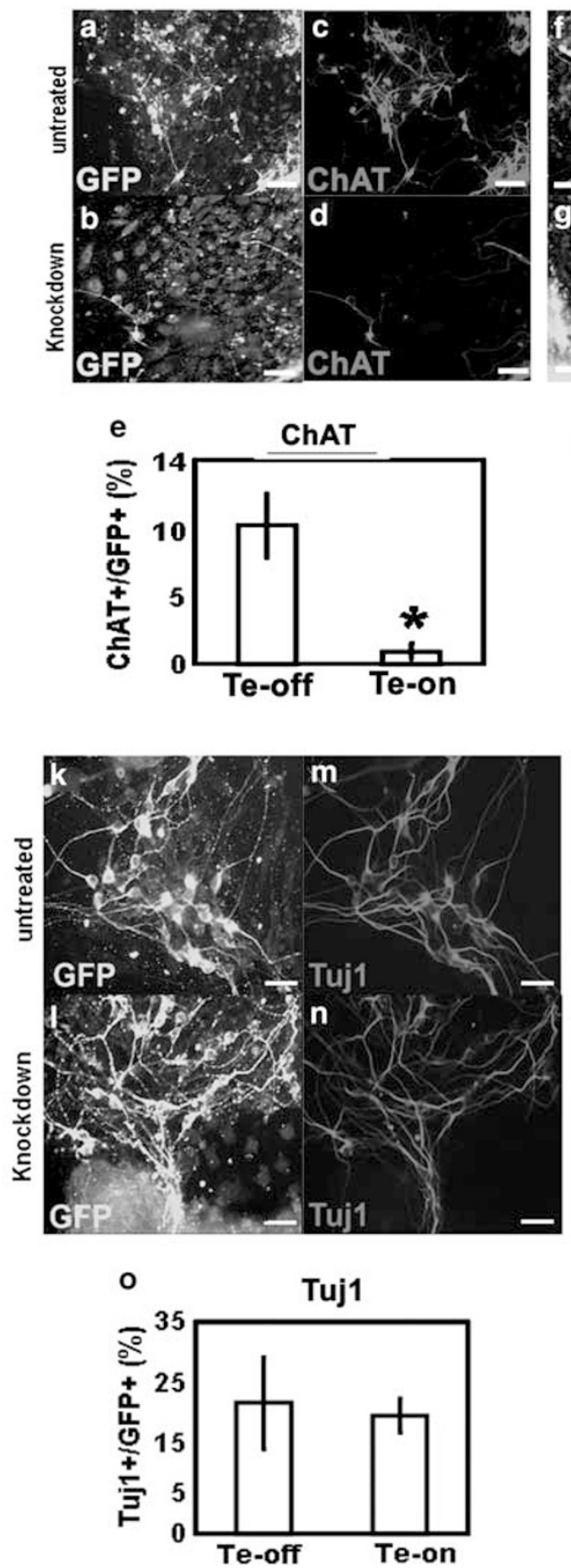

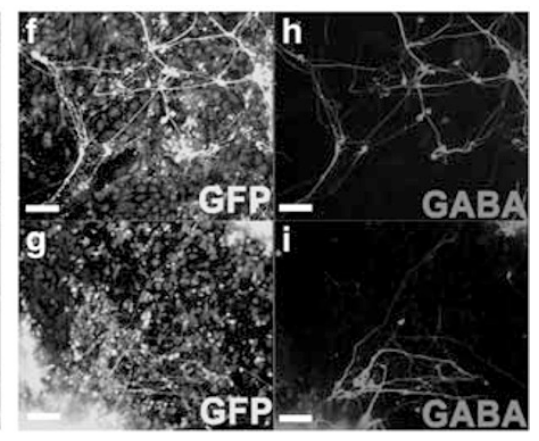

j
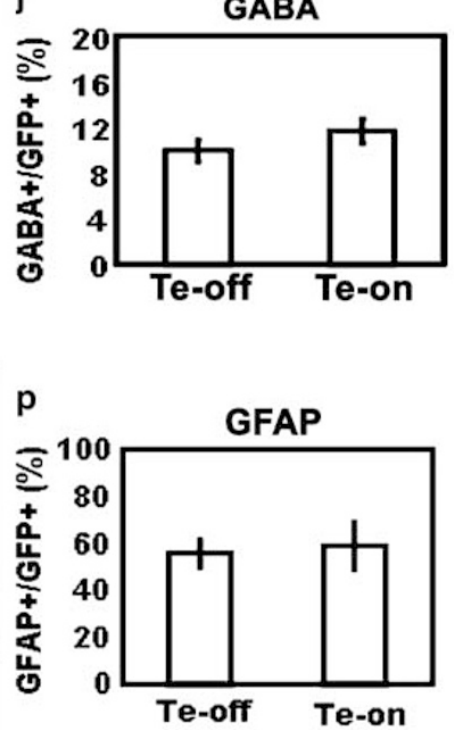

q

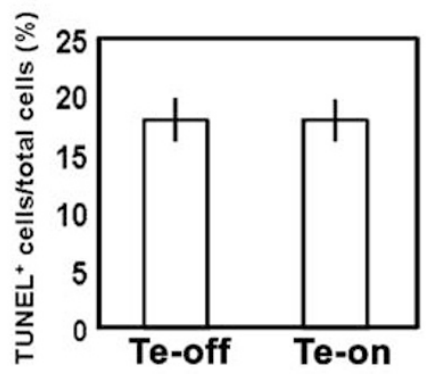

Figure 2 siRNA-mediated reduction of $L 3 / L h x 8$ expression decreases cholinergic differentiation of ES cells (single cells). (a-p) Spheres of ES cells were treated with trypsin, and then incubated in differentiation medium with (knockdown; Te-on; $\mathbf{b}, \mathbf{d}, \mathbf{g}, \mathbf{i}, \mathbf{l}, \mathbf{n}$ ) or without (untreated; Te-off; $\mathbf{a}, \mathbf{c}, \mathbf{f}, \mathbf{h}, \mathbf{k}, \mathbf{m}$ ) Te for 14 days. After incubation, the cells were fixed and immunostained with antibodies against GFP $(\mathbf{a}, \mathbf{b}, \mathbf{f}, \mathbf{g}, \mathbf{k}, \mathbf{l})$, ChAT $(\mathbf{c}, \mathbf{d}), \mathrm{GABA}(\mathbf{h}, \mathbf{i})$ or Tuj $\mathbf{1}(\mathbf{m}, \mathbf{n})$. Quantitative data from four separate experiments are shown as the percentages of ChAT- (e), GABA- (j), Tuj1- (o) or GFAP- (p) positive cells among the GFP-positive cells $(n=4)$. (q) Effects of Te-on or -off on cell death assay in ES cells. Quantitative data from three separate experiments are shown as the percentages of TUNEL-positive cells in the total cell number. Scale bar, $20 \mu \mathrm{m}$ 
in the Te-untreated (Te-off) cells (Figure 1e, upper panel; Figure 1f).

In our preliminary experiments, suppression of L3/Lhx8 expression by the Te-on system caused a reduction in the number of cholinergic projections from differentiated spheres (data not shown). In order to quantify these results, we treated spheres with trypsin to disperse the cells and performed immunofluorescence staining (Figure 2). Cholinergic differentiation was significantly decreased in the L3/Lhx8-knockdown Te-on ES cells compared to the Te-off cells (Figure 2a-e). However, no significant alterations were observed in the number of GABA-, Tuj1- or GFAP-positive cells between the Te-on and Te-off conditions (Figure $2 f-p)$. In addition, Tetreatment did not significantly influence the rate of cell death in the L3/Lhx8-knockdown clone (Figure $2 q$ ) or the number of processes (Te-off; $2.46 \pm 1.04$, Te-on; $2.43 \pm 0.96$ ).

In the present study, there was a significant reduction in the expression of a cholinergic marker in RA-treated ES cells after treatment with L3/Lhx8 siRNA (Figure 2a-e). The present results, together with those of our previous study, ${ }^{3}$ confirm that L3/Lhx8 positively regulates cholinergic differentiation. Although GABAergic differentiation was increased in differentiating L3/Lhx8-knockdown N2a cells, ${ }^{3}$ the number of ES cells positive for a GABAergic marker did not change significantly in this study (Figure $2 \mathrm{f}-\mathrm{j}$ ) and so the effect of L3/Lhx8 on GABAergic differentiation remains uncertain.

If $L 3 /$ Lhx8 is a key factor for cholinergic differentiation, overexpression of L3/Lhx8 alone would increase cholinergic marker expression and neuronal numbers. Moreover, the suppressed differentiation of cholinergic neurons mediated by L3/Lhx8 siRNA should be recovered by overexpression of L3/
Lhx8. In order to prove these hypotheses, we overexpressed the $L 3 / L h x 8$ gene at the time when differentiation appeared to begin. In L3/Lhx8-overexpressing cells, a greater number of cells were positive for the cholinergic marker ChAT (Figure $3 a-e)$, but there was no change in the number of GABAergic cells (Figure $3 \mathrm{f}$ ) or Tuj1 (Figure $3 \mathrm{~g}$ ), compared with control cells (transfection efficiency; $53.7 \% \pm 5.6$ ). In addition, there was no significant change in the rate of cell death following transfection of this clone (Figure $3 \mathrm{~h}$ ).

We went on to examine whether suppression of the cholinergic phenotype by L3/Lhx8 siRNA would be recovered by $L 3 /$ Lhx 8 overexpression. We found that exogenous expression of L3/Lhx8 was effective in offsetting the effects of L3/Lhx8 siRNA on ES cells (Figure $4 \mathrm{a}-\mathrm{g}$ ), while GABA- or Tuj1-positive cells remained unaffected by the recovery of L3/Lhx8 under the same conditions (Figure $4 \mathrm{~h}$ and i). No significant changes on cell death of this clone by Te-treatment or transfection were observed (Figure 4j). However, our previous study demonstrated that overexpression of L3/Lhx8 alone, in N2a cells, did not alter the RA-mediated differentiation to cholinergic or GABAergic neurons. ${ }^{3}$ One explanation for this discrepancy may be that the timing of $L 3 / L h \times 8$ expression may need to be adjusted to determine the action of L3/Lhx8 in the cholinergic differentiation process. In fact, judging by previous reports on the basic helix-loop-helix transcription factor Mash1 and the homeodomain transcription factor Dlx, which are important for neurogenesis in the basal forebrain at the early and late stages of development, respectively, ${ }^{18,19}$ the exact timing of transcription factor expression during developmental- and differentiation processes appears to be important. In any case, these results
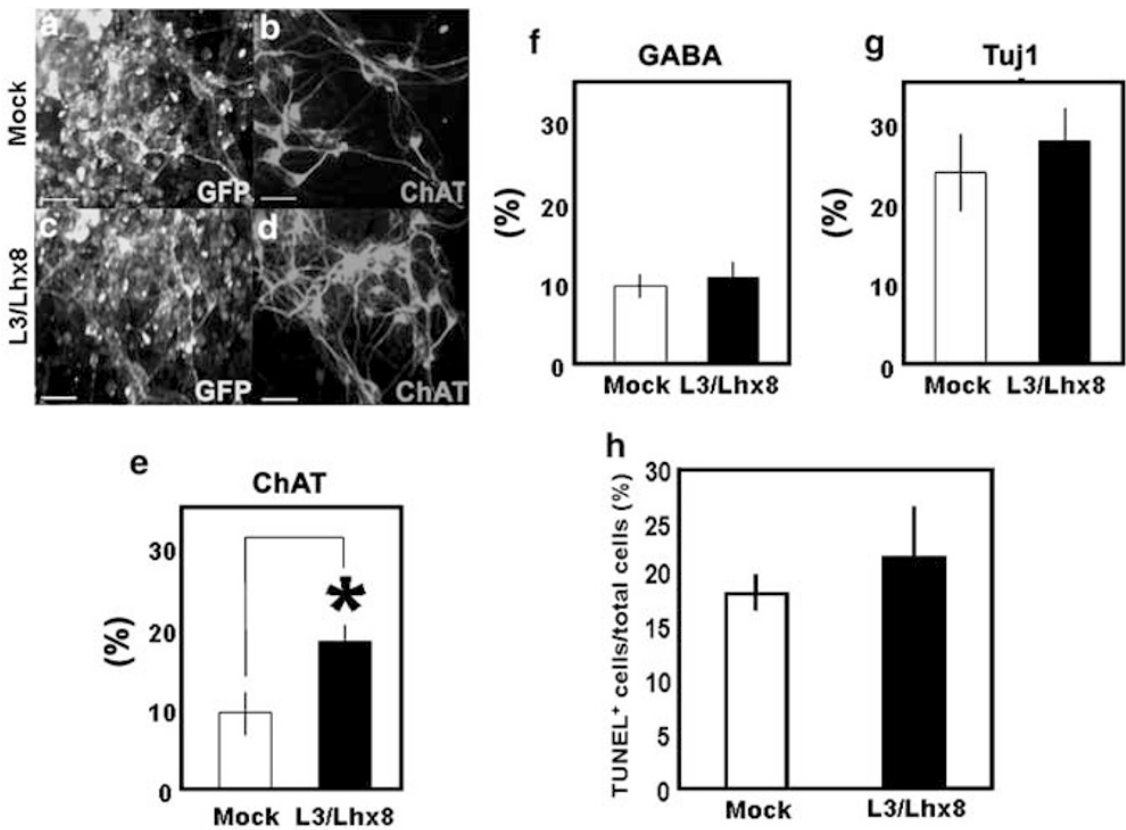

Figure 3 Effects of transient transfection of L3/Lhx8 on neural differentiation of ES cells. (a-g) Spheres of ES cells were treated with trypsin, and then seeded onto courting dishes. Eight hours after seeding out, the cells were transiently transfected with an $L 3 / L h x 8$ expression vector (c, d) or a mock vector (Mock; $\mathbf{a}, \mathbf{b})$ for $8 \mathrm{~h}$, and then incubated in differentiation medium for 14 days. After incubation, the cells were fixed and immunostained with antibodies against GFP (a, c) or ChAT (b, d). Quantitative data from four separate experiments are shown as the percentages of ChAT- (e), GABA- (f) or Tuj1- (g) positive cells among the GFP-positive cells $(n=4)$. ${ }^{*} P<0.05$. (h) Effect of transfecting cells with Mock or L3/Lhx8 on cell death assay in ES cells. Quantitative data from three separate experiments are shown as the percentages of TUNEL-positive cells in the total cell number. Scale bar, $20 \mu \mathrm{m}$ 


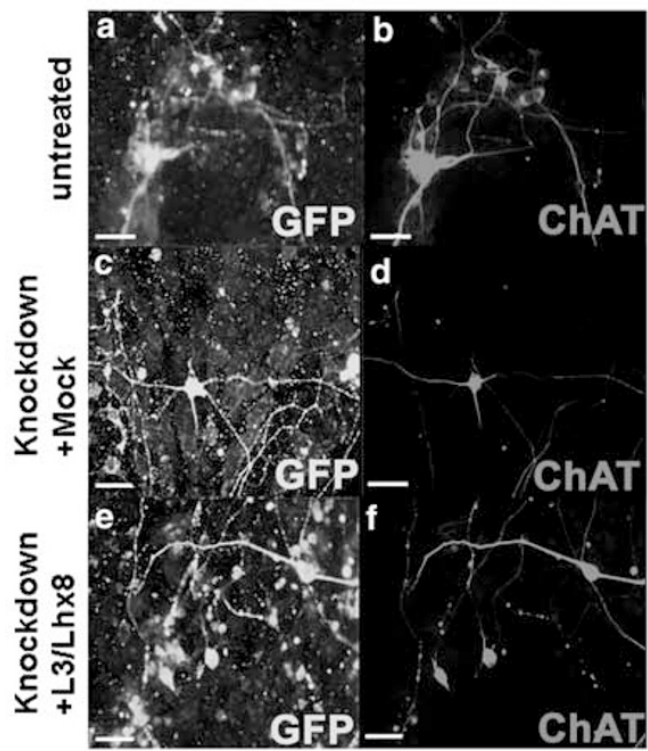

h
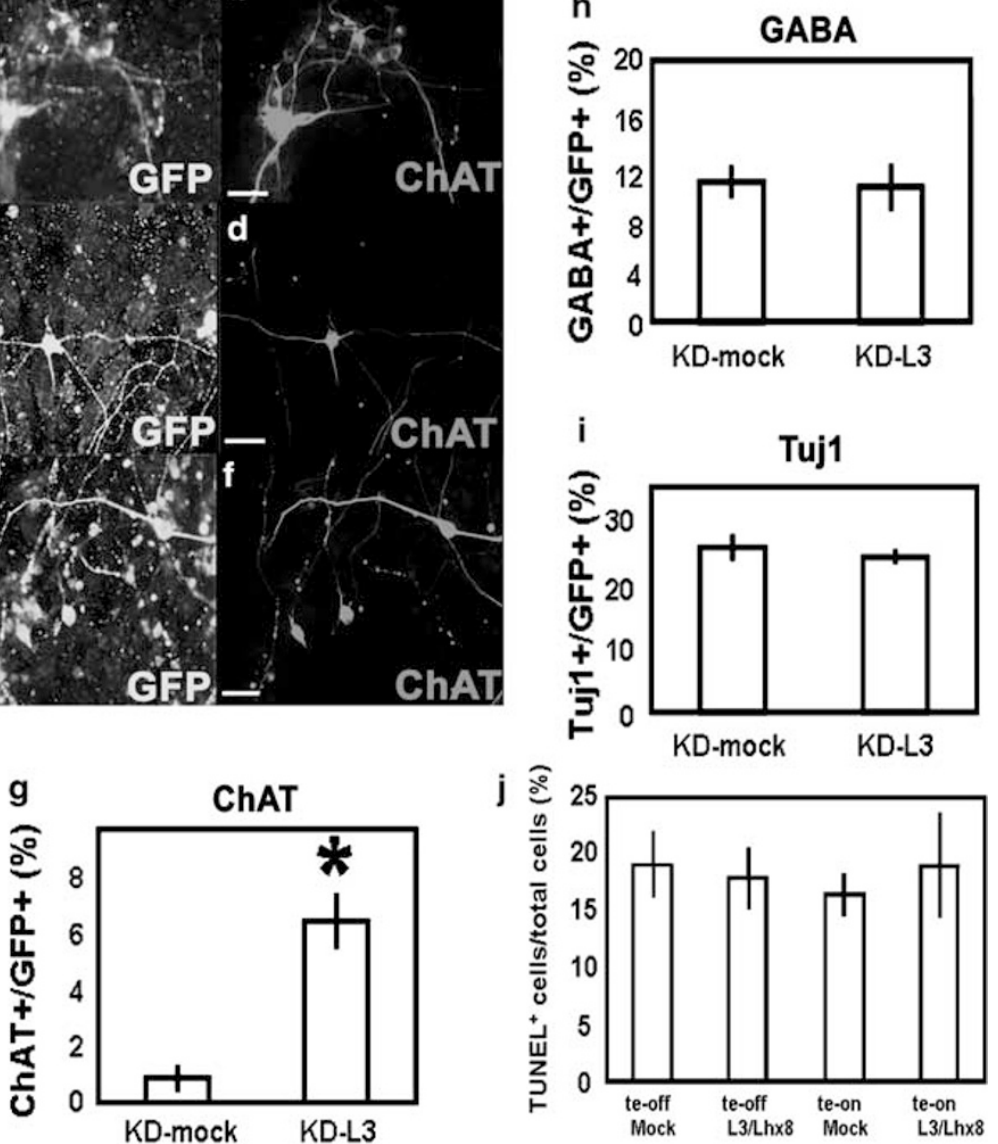

Figure 4 Effect of L3/Lhx8 expression on the L3/Lhx8 siRNA-induced decrease in cholinergic differentiation of ES cells. (a-i) Spheres of ES cells were treated with trypsin, and seeded onto courting dishes. Eight hours after seeding out, the cells were transiently transfected with an L3/Lhx8 expression vector (e, $\mathbf{f}$ ) or a mock vector (Mock; $\mathbf{c}, \mathbf{d}$ ) for $8 \mathrm{~h}$, followed by incubation in differentiation medium with (c-f) or without (a, b; untreated) Te for 14 days. After the treatments, the cells were fixed and immunostained with antibodies against GFP $(\mathbf{a}, \mathbf{c}, \mathbf{e})$ or ChAT $(\mathbf{b}, \mathbf{d}, \mathbf{f})$. Quantitative data from three separate experiments are shown as the percentages of ChAT- (g), GABA- (h) or Tuj1-(i) positive cells among the GFP-positive cells $(n=4)$. ${ }^{\star} P<0.05$. KD-mock; cells that were mock-transfected following knockdown of $\mathrm{L} 3 / \mathrm{Lh} \times 8$. KD-L3; cells that were $\mathrm{L} 3 / \mathrm{Lh} \times 8$-transfected following knockdown of L3/Lhx8. (j) Effects of Te-on or -off and transfection of Mock or L3/Lhx8 on cell death assay in ES cells. Quantitative data from three separate experiments are shown as the percentages of TUNEL-positive cells in the total cell number. Scale bar, $20 \mu \mathrm{m}$

strongly suggest that L3/Lhx8 plays a pivotal role in the specification of cholinergic neurons.

The results of the present study provide two important points of information. The first point is that L3/Lhx8 positively induces cholinergic specification of ES cells under normal conditions. This is the first study to use multipotent ES cells for this purpose and adds further proof to our previous results, which indicated that L3/Lhx8 plays a role in the development of cholinergic neurons in the basal forebrain. The second point is that L3/Lhx8 does not affect GABAergic specification of ES cells, in contrast to the requirements of the N2a cell line. ${ }^{3}$ To explain this discrepancy, two possible causes can be considered, namely the timing of L3/Lhx8 action and the participation of other factors. As mentioned above, N2a cells are pre-committed to a neuronal fate, and therefore the processes that regulate differentiation or the factors participating in these processes, may be simpler than those involved in the case of multipotent ES cells. In fact, many transcription factors besides L3/Lhx8, such as Lhx6, Isl-1, Gbx-1 and Gbx2 , are either sequentially or simultaneously involved in the differentiation of the basal forebrain, ${ }^{2,20}$ and this should be taken into account in future studies. In summary, the GABAergic phenotype may involve more complex underlying mechanisms in the ES cell paradigm; in order to elucidate these mechanisms, the interactions between L3/Lhx8 and the promoters of cholinergic and GABAergic marker genes should be examined.

\section{Materials and Methods}

Cell culture and differentiation. Mouse ES cells were maintained as monolayer cultures in growth medium consisting of $90 \%$ Dulbecco's modified eagle medium (DMEM), 10\% fetal bovine serum (FBS), $100 \mathrm{U} / \mathrm{ml}$ penicillin and $10 \mu \mathrm{g} / \mathrm{ml}$ streptomycin. The cells were incubated at $37^{\circ} \mathrm{C}$ in a humidified atmosphere of $95 \%$ air $/ 5 \% \mathrm{CO}_{2}$ and subcultured into fresh culture vessels when the growth reached $70-90 \%$ confluence (i.e. every $2-3$ days). For differentiation experiments, cells were plated on $35-\mathrm{mm}$ dishes at $1.5 \times 10^{5} \mathrm{cells} / \mathrm{dish}$ in FBS-/knockout serum replacement (KSR) + DMEM. Following incubation for $1 \mathrm{~h}$, the growth medium was replaced with FBS-/KSR + DMEM supplemented with $0.1 \mu \mathrm{M}$ RA. After incubation for the required length of time, the cells were harvested in homogenization buffer for RT-PCR or fixation buffer for immunocytochemistry. 
Vector construction. Vectors were constructed as described previously.,21

Transfections and stable clones. The pcDNA6/TR vector (Invitrogen) and pRNATin-H1.2 with L3/Lhx8 siRNA-inducible sequences were transfected into ES cells using Targefect F-1 (Targeting Systems). Individual clones were picked up after cell selection with hygromycin for pRNATin or Geneticin for pcDNA6/TR. Transient transfection of pcDNA3.1 $(+)$-L3/Lhx8 in to ES cells was also performed using Targefect F-1 while starting specialization conditions $(1 \mu \mathrm{g} / \mathrm{ml}$, transfection efficiency; $53.7 \% \pm 5.6)$.

Immunocytochemistry. Immunocytochemical analyses were performed as described previously. ${ }^{3,21}$ The primary antibodies used were anti-choline acetyltransferase (ChAT; mouse monoclonal; Chemicon), anti-GABA (rabbit polyclonal; Sigma) and anti-GFP (mouse monoclonal, Molecular Probes Inc.; rabbit polyclonal, Molecular Probes Inc.; goat polyclonal, Rockland).

Cell counting and data analysis. ES cells were cultured on Cellocate coverslips (Eppendorf). After double-label immunocytochemistry for GFP and cellular markers, the cells were observed under a fluorescence microscope. The total number of cells (100-150 cells in 3-4 different fields at 100-fold magnification) was counted using the GFP immunofluorescence to identify cells (labeled with an Alexa 688-conjugated secondary antibody (Molecular Probes Inc.) in a single experiment. Cells positive for the given marker were quantified under a fluorescence microscope with an appropriate filter set for Alexa 546 or Alexa 350 (Molecular Probes Inc.). The counting experiments were performed in triplicate for each marker. The results were expressed as the mean \pm S.E. and analyzed statistically by Student's $t$-test.

Real-time reverse-transcription-PCR. Total RNA was extracted and purified from ES cells under normal and differentiation conditions using a Trizol extraction method (Life Technologies). Reverse transcription (RT) of the purified RNA $(1 \mu \mathrm{g})$ was carried out for $1 \mathrm{~h}$ at $42^{\circ} \mathrm{C}$ in $50 \mu$ of Tris- $\mathrm{HCl}$ buffer $(50 \mathrm{mM}$, pH 8.3) containing $\mathrm{KCl}(75 \mathrm{mM}), \mathrm{MgCl}_{2}(3 \mathrm{mM})$, oligo(dT) $12-18(50 \mathrm{pmol})$ and deoxynucleotides $(200 \mu \mathrm{M})$ in the presence of murine Moloney leukemia virus reverse transcriptase (200 U; Promega). Polymerase chain reactions (PCR) were performed under the following conditions. The sense and antisense primers used for L3/Lhx8 were 5' $5^{\prime}$-ATGTATTGGAAGAGCGATCAG-3' and 5'-TCATTGGATGGG GTAACAAGGGC-3', respectively. A $1 \mu$ laliquot of the CDNA was used as the realtime PCR template in $20 \mu$ of reaction mixture containing the two primers. The amplified products were separated on $1 \%$ agarose gels and visualized by ethidium bromide staining and subsequent UV irradiation.

TUNEL assay. Cells were processed for terminal deoxynucleotidyl transferase mediated dUTP nick end labeling (TUNEL) by using an in situ cell death detection kit (Roche) following the protocol provided by the manufacturer.

Acknowledgements. We thank Dr. H Niwa (RIKEN) for providing the murine ES cells. TM was supported by a grant from The Ichiro Kanehara Foundation. The present study was supported in part by a Grant-in-Aid for Scientific Research on Priority Areas, Advanced Brain Science Project, from the Ministry of Education, Culture, Sports, Science and Technology, Japan.
1. Matsumoto $\mathrm{K}$, Tanaka T, Furuyama T, Kashihara $\mathrm{Y}$, Mori $\mathrm{T}$, Ishii $\mathrm{N}$ et al. L3, a novel murine LIM-homeodomain transcription factor expressed in the ventral telencephalon and the mesenchyme surrounding the oral cavity. Neurosci Lett 1996; 204: 113-116.

2. Mori T, Yuxing Z, Takaki H, Takeuchi M, Iseki K, Hagino S et al. The LIM homeobox gene, $\mathrm{L} 3 / \mathrm{Lhx}$, is necessary for proper development of basal forebrain cholinergic neurons. Eur $J$ Neurosci 2004; 19: 3129-3141.

3. Manabe T, Tatsumi K, Inoue M, Matsuyoshi H, Makinodan M, Yokoyama S et al. L3/Lhx8 is involved in the determination of cholinergic or GABAergic cell fate. J Neurochem 2005; 94 : 723-730.

4. Whitehouse PJ, Price DL, Clark AW, Coyle JT, DeLong MR. Alzheimer disease: evidence for selective loss of cholinergic neurons in the nucleus basalis. Ann Neurol 1981; 10: 122-126.

5. Whitehouse PJ, Price DL, Struble RG, Clark AW, Coyle JT, Delon MR. Alzheimer's disease and senile dementia: loss of neurons in the basal forebrain. Science 1982; 215: 12371239 .

6. Kawasaki H, Mizuseki K, Nishikawa S, Kaneko S, Kuwana Y, Nakanishi S et al. Induction of midbrain dopaminergic neurons from ES cells by stromal cell-derived inducing activity. Neuron 2000; 28: 31-40.

7. Niwa H, Miyazaki J, Smith AG. Quantitative expression of Oct-3/4 defines differentiation, dedifferentiation or self-renewal of ES cells. Nat Genet 2000; 24: 372-376.

8. Shimozaki K, Nakashima K, Niwa H, Taga T. Involvement of Oct3/4 in the enhancement of neuronal differentiation of ES cells in neurogenesis-inducing cultures. Development 2003; 130: 2505-2512.

9. Zeller M, Strauss WL. Retinoic acid induces cholinergic differentiation of NTera 2 human embryonal carcinoma cells. Int J Dev Neurosci 1995; 13: 437-445.

10. Pedersen WA, Berse B, Schuler U, Wainer BH, Blusztain JK. All-trans- and 9-cis-retinoic acid enhance the cholinergic properties of a murine septal cell line: evidence that the effects are mediated by activation of retinoic acid receptor-alpha. J Neurochem 1995; 65: $50-58$.

11. Hill DP, Robertson KA. Characterization of the cholinergic neuronal differentiation of the human neuroblastoma cell line LA-N-5 after treatment with retinoic acid. Brain Res Dev Brain Res 1997; 102: 53-67.

12. Kornyei $Z$, Toth $B$, Tretter $L$, Madarasz E. Effects of retinoic acid on rat forebrain cells derived from embryonic and perinatal rats. Neurochem Int 1998; 33: 541-549.

13. Malik MA, Greenwood CE, Blusztajn JK, Berse B. Cholinergic differentiation triggered by blocking cell proliferation and treatment with all-trans-retinoic acid. Brain Res 2000; 874: 178-185.

14. Personett D, Fass U, Panickar K, McKinney M. Retinoic acid-mediated enhancement of the cholinergic/neuronal nitric oxide synthase phenotype of the medial septal SN56 clone: establishment of a nitric oxide-sensitive proapoptotic state. J Neurochem 2000; 74: 2412 2424.

15. Fischer HS, Berti I, Schatz DS, Humpel C, Saria A. Retinoic acid treatment enhances the acetylcholine contents in the human teratocarcinoma cell line NTera-2. Regul Pept 2000; 96: 59-63.

16. Wichterle H, Lieberam I, Porter JA, Jessell TM. Directed differentiation of embryonic stem cells into motor neurons. Cell 2002; 110: 385-397.

17. Chu PW, Cheung WM, Kwong YL. Differential effects of 9-cis, 13-cis and all-trans retinoic acids on the neuronal differentiation of human neuroblastoma cells. Neuroreport 2003; 14 : 1935-1939.

18. Yun K, Fischman S, Johnson J, Hrabe de Angelis M, Weinmaster G, Rubenstein JL. Modulation of the notch signaling by Mash1 and Dlx1/2 regulates sequential specification and differentiation of progenitor cell types in the subcortical telencephalon. Development 2002; 129: 5029-5040.

19. Marin $\mathrm{O}$, Anderson SA, Rubenstein JL. Origin and molecular specification of striatal interneurons. J Neurosci 2000; 20: 6063-6076.

20. Asbreuk CH, van Schaick HS, Cox JJ, Kromkamp M, Smidt MP, Burbach JP. The homeobox genes Lhx7 and Gbx1 are expressed in the basal forebrain cholinergic system. Neuroscience 2002; 109: 287-298.

21. Manabe T, Katayama T, Sato N, Gomi F, Hitomi J, Yanagita T et al. Induced HMGA1a expression causes aberrant splicing of Presenilin-2 pre-mRNA in sporadic Alzheimer's disease. Cell Death Differ 2003; 10: 698-708. 